\title{
In-utero Three Dimension High Resolution Fetal Brain Diffusion Tensor Imaging
}

\author{
Shuzhou Jiang ${ }^{1}$, Hui Xue ${ }^{1,2}$, Serena Counsell ${ }^{1}$, Mustafa Anjari ${ }^{1}$, Joanna Allsop ${ }^{1}$, \\ Mary Rutherford ${ }^{1}$, Daniel Rueckert ${ }^{2}$, and Joseph V. Hajnal ${ }^{1}$ \\ ${ }^{1}$ Imaging Sciences Department, MRC Clinical Sciences Centre, Hammersmith Hospital, \\ Imperial College London, London, United Kingdom \\ ${ }^{2}$ Department of Computing, Imperial College London, London, United Kingdom
}

\begin{abstract}
We present a methodology to achieve 3D high resolution in-utero fetal brain DTI that shows excellent ADC as well as promising FA maps. After continuous DTI scanning to acquire a repeated series of parallel slices with 15 diffusion directions, image registration is used to realign the images to correct for fetal motion. Once aligned, the diffusion images are treated as irregularly sampled data where each voxel is associated with an appropriately rotated diffusion direction, and used to estimate the diffusion tensor on a regular grid. The method has been tested successful on eight fetuses and has been validated on adults imaged at $1.5 \mathrm{~T}$.
\end{abstract}

\section{Introduction}

Fetal brain imaging by MRI is attracting increasing interest because it offers excellent contrast and anatomical detail. However, unpredictable fetal motion has led to the widespread use of single shot techniques that can freeze fetal motion for individual slices. An even greater challenge is in-utero Diffusion Weighted Imaging (DWI). Preliminary trials $[1 ; 2]$ have been performed in which for a few relatively thick slices, $a b_{0}$ image and diffusion weighted images with 3 directions of sensitization were acquired within a maternal breath hold time. These early experiments relied on the chance event of the fetus remaining still for all 4 sets of images so that apparent diffusion coefficients (ADC) could be calculated. Diffusion tensor imaging (DTI) offers the potential for more information than DWI particularly for tractography studies. However, DTI is even more challenging than DWI for fetal imaging [3], because it requires a $b_{0}$ image and at least 6 diffusion images that are sensitized in non-collinear directions for each slice studied. These extra images increase the minimum acquisition time so that the requirements for the maternal breath-hold become more onerous. Without a maternal breath-hold fetal motion combines with the mother's respiration to disrupt the spatial correspondence between component images required to calculate tensor properties.

Snapshot imaging with Volume Reconstruction (SVR) is a method developed to perform 3D high resolution and high SNR in-utero anatomical imaging of the fetal brain using dynamic scanning and image registration[4-6]. In this study we extended the SVR technique to DTI of the in-utero fetal brain and validated it using adult data. 


\section{Method and Implementation}

We will restrict the discussion to structures that can be treated as rigid bodies undergoing an unknown motion and shall take the fetal brain as an exemplar of such structures. The scenario of interest involves motion that is fast enough to preclude self consistent whole brain imaging even with echo planar imaging (EPI) (typical time $>3$ seconds), but slow enough to produce individual high quality 2D images (typical time $<100 \mathrm{msec})$. The acquired slices consist of voxels which have a measured intensity $\mathrm{I}(\mathrm{x}, \mathrm{y}, \mathrm{z})$ at prescribed positions in the scanner frame of reference, $\Omega_{0}(\mathrm{x}, \mathrm{y}, \mathrm{z})$. We now adopt a $3 \mathrm{D}$ Cartesian coordinate system $\Omega(\mathrm{x}, \mathrm{y}, \mathrm{z})$ fixed relative to the anatomy of interest. This coordinate frame moves relative to the scanner coordinate frame as the subject moves. The acquired voxel data are legitimate samples in $\Omega$, but at unknown locations $(\mathrm{x}, \mathrm{y}, \mathrm{z})$. Because of the motion, the samples $\mathrm{I}(\mathrm{x}, \mathrm{y}, \mathrm{z})$ are irregularly spaced in $\Omega$. Provided their locations can be determined and the samples are sufficiently dense throughout the region of interest in $\Omega$, data interpolation can be used to generate a full representation on a regular Cartesian lattice in $\Omega$. In the case of DTI data, the individual diffusion weighted slices have different contrast both relative to the $b_{0}$ images and to each other because of the effects of varying the direction of sensitization on anisotropic tissues such as white matter tracts. In addition the correct sensitization direction for each acquired slice must be maintained once it is correctly placed in the anatomical space. Successful reconstruction of a 3D representation of diffusion tensor matrix that can then be reformatted into any desired plane thus has 3 requirements:

1) Sufficient samples in $\Omega$ with at least 6 independent diffusion directions as well as a non-diffusion weighted $(b=0)$ image to allow full representation of the anatomical structure.

2) Determination of the mapping from $\Omega_{0}$ to $\Omega$ for each sample

3) Reconstruction of the diffusion tensor matrix D on a regular sampled 3D space given the scattered, irregularly spaced samples $\mathrm{I}(\mathrm{x}, \mathrm{y}, \mathrm{z})$ that are each associated with an appropriately oriented diffusion gradient direction.

\subsection{Achieving the Required Sample Density}

In the proposed application the fetal brain to be imaged may move between acquisition of individual slice acquisitions. This causes uneven slice samples when viewed in the anatomical reference frame $(\Omega)$ and is likely to result in violation of the Nyquist sampling criterion at some locations. To avoid this problem the target volume in $\Omega_{0}$ is repeatedly imaged by simply looping through all slice positions so that in the absence of motion each location is sampled with at least 6 different diffusion gradients multiple times. We refer to each complete set of slices spanning $\Omega_{0}$ for the entire set of diffusion weighted images for different diffusion gradient directions plus one non diffusion weighted image as a loop. The number of loops required depends on the motion. In these experiments we used 3-4 loops each with 15 non-linear diffusion directions subject to the total scan duration being acceptable to the pregnant subjects. 


\subsection{Motion Compensation Through Slice to Volume Registration}

A self consistent $3 \mathrm{D} \mathrm{b}_{0}$ image $\mathrm{V} 0$ is first reconstructed using the method described in $[5 ; 6]$. Then each diffusion weighted slice is registered to the $\mathrm{b}_{0}$ volume $\mathrm{V} 0$ using normalized mutual information (NMI)[7] as the cost function. Registration proceeds in a temporally hierarchical manner as follows: The data is first divided into temporally contiguous blocks each containing multiple slices that together provide full coverage of the volume of $\Omega_{0}$ of interest (i.e. each loop is divided into 15 stacks of diffusion weighted images). Initially subject motion between slices in a stack is ignored, these stacks of slices are treated as $3 \mathrm{D}$ volumes and registered to the $3 \mathrm{D} \mathrm{b}_{0}$ volume $\mathrm{V} 0$ using rigid body transformations.

Once the data is aligned, the size of temporally contiguous blocks is reduced so the data is divided into sub-packages that are temporally contiguous although the slices in each sub-package may not be spatially contiguous. These sub-packages are each registered to V0. The registration process involves moving the sub-package with V0 held fixed in space, but always interpolating the V0 intensity values to provide comparison intensity values. When the transformations $\left(\mathrm{T}\left(\Omega_{0} \rightarrow \Omega\right)\right.$ ) of all subpackages have been determined, the time scale is reduced again and the process repeated until each slice is treated in isolation.

Followed the method in[5;6;8], a slice/stack-to-volume registration method based on a multi-start, multi-resolution approach using the Powell method to optimize each transformation parameter in turn was developed.

\subsection{Reconstruction of the Diffusion Tensor Matrix}

Using an anatomic coordinate system fixed relative to the reconstructed $\mathrm{b}_{0}$ image volume $\mathrm{V} 0$, and given acquired diffusion sensitization direction $\mathrm{g}$, rotation matrix $\mathrm{R}$ with respect to $\mathrm{V} 0$, and the corresponding intensity $I_{0}$ in $\mathrm{V} 0$ determined via cubic Bspline interpolation [9], the diffusion intensity $I$ can be determined by:

$$
I=I_{0} * \exp \left(-b g^{\prime} D_{L a b} g\right)=I_{0} * \exp \left(-b g^{\prime}\left(R^{\prime} D_{A n a} R\right) g\right)
$$

$D_{L a b}$ and $D_{A n a}$ are the diffusion tensor matrix in laboratory and anatomic coordinate respectively, $g$ is a unit vector in the direction of sensitization and $b$ is the diffusion sanitization parameter in $\mathrm{s} / \mathrm{mm}^{2}$. We can further obtain a normalized logarithm value $\mathrm{s}$ using eq.(2) to prepare for later linear tensor fitting.

$$
s=-\log \left(I / I_{0}\right) / b=g^{\prime}\left(R^{\prime} D_{\text {Ana }} R\right) g
$$

Once aligned, we treat the data s as irregularly sampled with each voxel associated with an appropriately rotated diffusion direction, and use all the data to estimate the diffusion tensor D on a regular grid. For each scattered point $s_{\text {satter }}$, we can use (3) to define its value, where the $\beta_{i}$ are spatial coefficients associated with regularly sampled diffusion tensor matrix $D_{\text {Ana; regular }, i}$.

$$
s_{\text {scatter }}=g^{\prime}\left(R^{\prime} D_{\text {Ana;scatter }} R\right) g=\sum_{i=1}^{N} \beta_{i} g^{\prime}\left(R^{\prime} D_{\text {Ana; regular }, i} R\right) g
$$

We can then reconstruct the scattered diffusion tensor to a regular grid by solving a huge matrix equation, (4),

$$
\bar{S}_{\text {scatter }}=M_{R} M_{G} * \operatorname{diag}\left(M_{S}, M_{S}, M_{S}, M_{S}, M_{S}, M_{S}\right) *\left[D_{x x} ; D_{y y} ; D_{z z} ; D_{x y} ; D_{x z} ; D_{y z}\right]
$$


where $\bar{S}_{\text {scatter }}$ is a vector containing all the scattered points, $M_{R}$ is a matrix specifying rotations $\mathrm{R}, M_{G}$ contains the diffusion gradient directions and $M_{S}$ is a spatial interpolation matrix. They are all sparse. $D_{x x}-D_{y z}$ are 6 independent parameters of the diffusion tensor. We use the Sparse Equations and Least SQuares Regression (LSQR)[10] method to solve for sparse matrix $D_{x x}-D_{y z}$, and then calculate its eigenvalues at each grid point to determine corresponding ADC and FA values. Nearest neighbor interpolation is used here to estimate the spatial coefficient $\beta_{i}$ given dense sampling.

The $3 \times 3$ symmetric diffusion tensor matrix $\mathrm{D}$ should be positive definite. A further refinement using constraint minimization is performed to guarantee the positive definition of the diffusion matrix.

\subsection{Fetal Scanning Protocol}

Fetal brain images were acquired on a $1.5 \mathrm{~T}$ Philips Achieva scanner (Best, The Netherlands) using a 5 channel torso array with a spin echo EPI diffusion tensor sequence. Image matrix of $150 \times 150$, field of view of $300 \mathrm{~mm}$ and slice thickness 3 to $4 \mathrm{~mm}$ with slices centered every half slice thickness, i.e., 1.5 to $2 \mathrm{~mm}$ were used depending on the maturity of the fetus. 60 slices covered the entire region of fetal brain. Slices were acquired in 2 packages and within each packages starting at one extreme end in an interleaved order to avoid slice cross-talk. We set the repeat time TR to be $12 \mathrm{~s}$ to avoid spin history effects while TE is chosen to be the shortest possible $(54 \mathrm{~ms})$ for the chosen b-value of $500 \mathrm{~s} / \mathrm{mm}^{2}$. Typical scanning time was 7 minutes for each loop of 15 diffusion directions with the SAR lower than $0.5 \mathrm{~W} / \mathrm{kg}$. These studies were approved by Hammersmith Hospital Ethics Committee.

\section{Verification Using Adult Data}

Images of adult brains acquired with spin echo EPI were used to test the accuracy of slice-to-volume registration for diffusion data and the subsequent reconstruction. Two datasets were acquired using the same diffusion tensor imaging protocol at $1.5 \mathrm{~T}$ but with a SENSE head coil. Slice-to-volume registration accuracy is validated using similar experiment described in [4;5]. 819 single slices from both diffusion and nondiffusion weighted scans of the same subject were selected, and each was registered to the $b_{0}$ volume and compared with the gold standard volume-to-volume registration. The slice-to-volume registration was found to be accurate within $1 / 4$ voxel size.

In a second set of experiments an adult volunteer deliberately moved about once per second, including making head rotations around all three axis up to 30 degree. 4 loops of 15 direction DTI data were acquired with in-plane resolution $3 \mathrm{~mm} \times 3 \mathrm{~mm}$, slice thickness $3.6 \mathrm{~mm}$ and $-1.8 \mathrm{~mm}$ slice gap. TR was 8 seconds while TE was $55 \mathrm{~ms}$ which was the minimum for a b value $500 \mathrm{~s} / \mathrm{mm}^{2}$. For comparison, images were also acquired with the same protocol while the subject was still. All the slices that were not corrupted by in-plane motion were registered and reconstructed. Motion effects in the diffusion tensor data were easily identified because these generally caused 


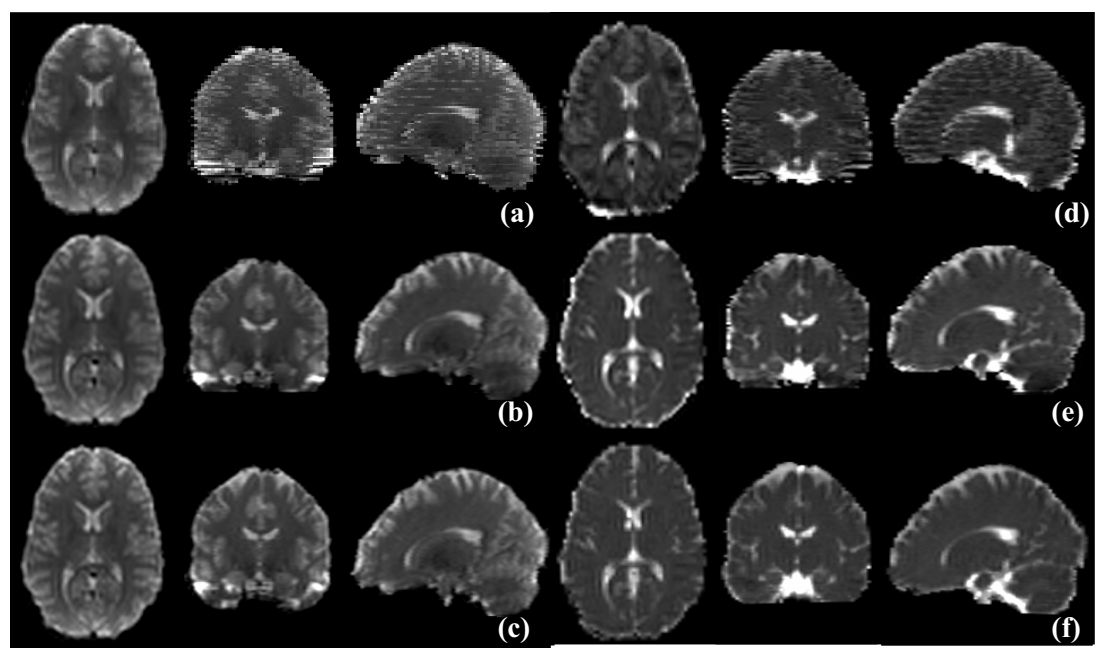

Fig. 1. Results for adult volunteer that deliberately moved during scanning: (a) is one loop of acquired transverse $b=0$ slices viewed in $3 \mathrm{D},(\mathrm{d})$ is the reconstructed $\mathrm{ADC}$ map before motion correction. (b) and (e) are reconstructed $b=0$ volume and ADC map. (c) and (f) are corresponding volume from stationary gold standard.

catastrophic reconstruction failures with gross signal loss in part of the individual EPI slices. The results were then compared to the gold standard (stationary volunteer) data to evaluate the method.

For the moving subject DTI data, reconstruction fidelity was assessed visually using difference images and by calculating the mean absolute difference and its standard deviation (STD) between the gold standard and the reconstruction for $b_{0}$ volume, ADC and FA values. Region of Interest (ROI) measurements of both ADC and FA values are also performed.

A self-consistent 3D $b_{0}$ image volume was first reconstructed using the SVR method [5]. As displayed in Fig 1, the reconstructed B0 data is almost identical to the gold standard $b_{0}$ data that was acquired when the subject stayed still. The standard error, i.e., the square root of the residual mean square, between the reconstructed volume and the gold standard is $3.6 \%$.

After motion correction and fitting of the diffusion tensor to the resulting scattered data, the reconstructed 3D ADC map (Fig. 1 (e)) shows clear consistency of ADC values. This contrasts with the corrupted ADC evaluation obtained without motion correction (Fig 1. (d)). When compared to the gold standard ADC values of the same subject, the result had a standard error of 5.6\%. The 3D FA map as displayed in Fig. 2 (b) is quite close to the gold standard displayed in Fig. 2 (c) as well, and was found to achieve a standard error of $6.7 \%$.

Furthermore, 8 Regions of Interest (ROI) measurement including Genu of Corpus Callosum (GCC), Splenium of Corpus Callosum (SCC), Posterior Limb of Internal Capsule (PLIC), Forceps major (FC), Anterior Limb of Internal Capsule (ALIC), Posterior region of Corona Radiate (PCR), Superior Longitudinal Fasciculus (SLF) 
and Superior region of Corona Radiate (SCR) on both ADC and FA values as displayed in Fig. 2(c) were performed for detailed validation. The reconstructed ADC and FA values were in excellent agreement with the gold standard (still subject) data in all 8 ROIs as listed in Table 1 and 2.

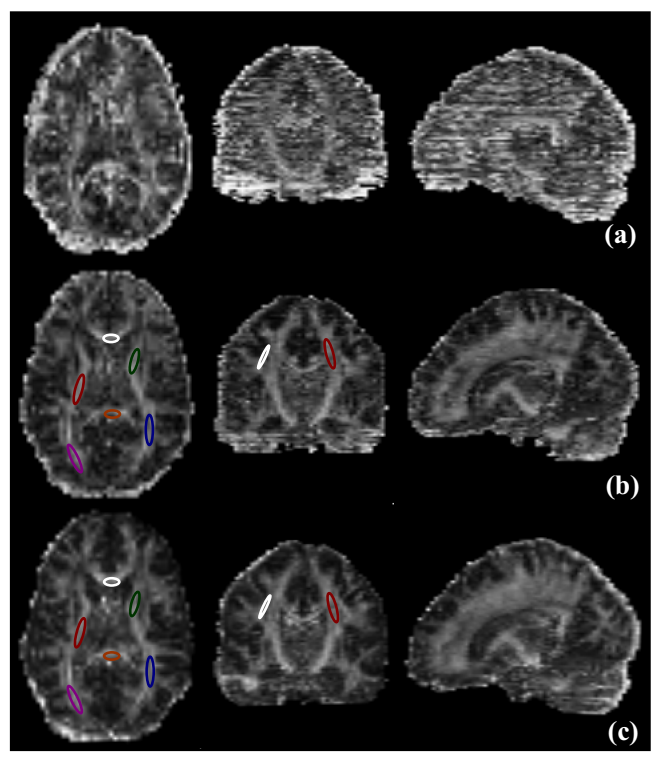

Fig. 2. Results for adult volunteer that deliberately moved during scanning: FA maps. (a) is the FA map reconstructed directly before motion correction. (b) are the corresponding views of the FA maps reconstructed to a $3 \mathrm{~mm} \times 3 \mathrm{~mm} \times 1.8 \mathrm{~mm}$ resolution image after registration and scattered diffusion tensor fitting. (c) are the corresponding views of the FA maps from the gold standard data from the stationary volunteer. 8 Regions of Interest (ROI) measurements on the ADC and FA maps from both the reconstructed data and the gold standard stationary subject data were performed. The ROI include samples in the GCC(while), SCC(orange), PLIC(red), FC(purple), ALIC(green) and PCR(blue) on the transverse view and SLF(white) and SCR(red) on the coronal orientation.
Table 1. ROI Measurement on ADC

\begin{tabular}{|l|l|l|}
\hline & Rec ADC & GS ADC \\
\hline SCC & $0.75 \pm 0.05$ & $0.78 \pm 0.11$ \\
\hline PLIC & $0.88 \pm 0.09$ & $0.91 \pm 0.06$ \\
\hline FC & $0.88 \pm 0.09$ & $0.88 \pm 0.09$ \\
\hline GCC & $0.89 \pm 0.09$ & $0.91 \pm 0.10$ \\
\hline ALIC & $0.80 \pm 0.07$ & $0.80 \pm 0.08$ \\
\hline PCR & $0.86 \pm 0.04$ & $0.84 \pm 0.04$ \\
\hline SLF & $0.81 \pm 0.06$ & $0.83 \pm 0.05$ \\
\hline SCR & $0.84 \pm 0.05$ & $0.86 \pm 0.04$ \\
\hline
\end{tabular}

Table 2. ROI Measurement on FA $\left(\mu m^{2} / m s\right)$

\begin{tabular}{|l|l|l|}
\hline & Rec FA & GS FA \\
\hline SCC & $0.74 \pm 0.11$ & $0.75 \pm 0.11$ \\
\hline PLIC & $0.64 \pm 0.04$ & $0.63 \pm 0.04$ \\
\hline FC & $0.56 \pm 0.09$ & $0.57 \pm 0.11$ \\
\hline GCC & $0.65 \pm 0.09$ & $0.63 \pm 0.07$ \\
\hline ALIC & $0.56 \pm 0.07$ & $0.56 \pm 0.09$ \\
\hline PCR & $0.56 \pm 0.09$ & $0.58 \pm 0.11$ \\
\hline SLF & $0.52 \pm 0.10$ & $0.53 \pm 0.10$ \\
\hline SCR & $0.42 \pm 0.07$ & $0.42 \pm 0.04$ \\
\hline
\end{tabular}

\section{Results}

Eight fetuses with gestational age between 24 and 34 weeks as listed in Table 3 and 4 were examined. Data from all the examinations have been successfully reconstructed. 


\subsection{Fetal Examples}

Fig. 3 shows data from a fetus of gestational age 26 weeks 5 days that had enlarged ventricles and agenesis of the corpus callosum. It was scanned with 15 diffusion gradient directions, and 4 loops of transverse images were acquired with $2 \mathrm{~mm}$ inplane resolution and $4 \mathrm{~mm}$ ( $2 \mathrm{~mm}$ slice separation) slice thickness. Approximately $25 \%$ of slices were corrupted because of extreme sensitivity to motion, and so had to be excluded. The acquired slice data is not consistent in space Fig. 3 (a). Final reconstruction achieved $2 \mathrm{~mm}$ isotropic resolution in all three dimensions for $\mathrm{b}=0$ images, as well as ADC and FA maps. Successful reconstruction of the $3 \mathrm{D} b=0$ image volume shown in Fig. 3 (b) provides an accurate target for registering the diffusion weighted slices. After motion correction, the reconstructed 3D ADC map Fig. 3 (c) shows clearly consistent appearances of ADC values. Different tissue types can clearly be differentiated, i.e. WM, CSF and cortex on the FA map Fig. 3 (d). The FA calculation is less robust than ADC and the results show greater fluctuations. A high resolution anatomical volume acquired with T2-W single short Turbo Spin Echo (ssTSE) dynamic sequence was also reconstructed for comparison in Fig. 3 (e) with $1.25 \mathrm{~mm}$ cubic resolution.

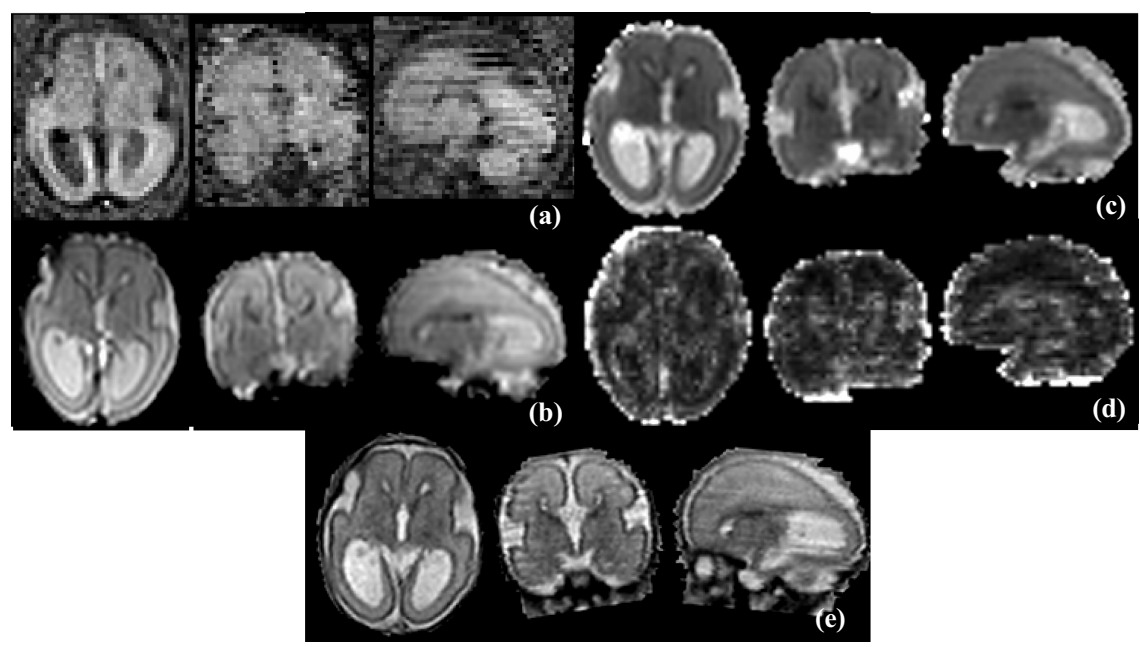

Fig. 3. 15-direction DTI of a fetus of 26 weeks plus 5 days. (a) is one loop of acquired fetal Diffusion transverse data viewed in Transverse, Coronal and Sagittal planes with $2 \mathrm{~mm} \times 2 \mathrm{~mm}$ in plane resolution, $4 \mathrm{~mm}$ slice thickness and -2mm gap. (b)-(d) are corresponding reconstructed b0 image, ADC map and FA map respectively with $2 \mathrm{~mm}$ cubic resolution. (e) is reconstructed anatomical data.

\subsection{ROI Analysis for ADC and FA Values}

Region of Interest (ROI) analysis was performed on all fetal subjects for 9 regions as shown in Table 3 and 4. ADC measurements could be made for all 8 subjects. 5 of them had FA maps of sufficient quality for measurement; while the rest 3 have much 

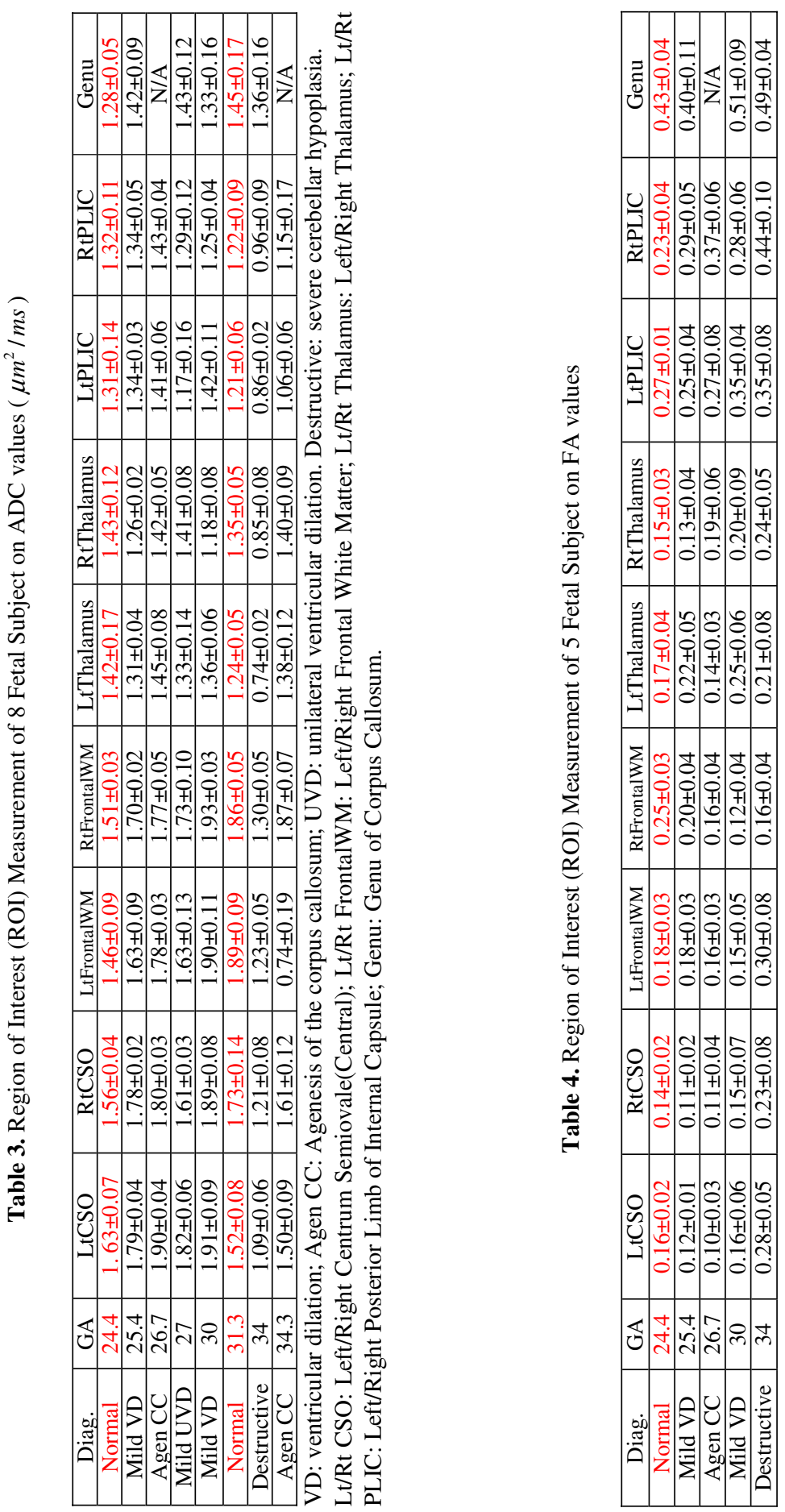
less good quality diffusion weighted raw images caused by severe motion to support robust FA calculation. Both ADC and FA have been shown to be age dependent in premature infants and to be changed by pathology [11]. Our fetal data, especially the normal subjects, are consistent with these ex-utero results.

\section{Conclusion}

We have described a methodology for diffusion tensor imaging of moving subjects by combining registered single shot $2 \mathrm{D}$ slices from sequential diffusion weighted scans. The method has been performed successfully on adults and especially on fetuses, for which no effective conventional diffusion tensor imaging method is currently available. The results allow fine structure of both ADC and FA maps to be clearly revealed potentially providing a means of monitoring microstructural development of the in-utero fetal brain for the first time. A future development that we would like to pursue is tractography of the fetal brain.

\section{References}

[1] Righini, A., et al.: Apparent diffusion coefficient determination in normal fetal brain: a prenatal MR imaging study. AJNR 24, 799-804 (2003)

[2] Prayer, D., et al.: MRI of normal fetal brain development. Eur.J.Radiol. 57(2), 199-216 (2006)

[3] Bui, T., et al.: Microstructural development of human brain assessed in utero by diffusion tensor imaging. Pediatr Radio 36, 1133-1140 (2006)

[4] Rousseau, F., et al.: A novel approach to high resolution fetal brain MR imaging. In: Duncan, J.S., Gerig, G. (eds.) MICCAI 2005. LNCS, vol. 3749, pp. 548-555. Springer, Heidelberg (2005)

[5] Jiang, S., et al.: A novel approach to accurate 3D high resolution and high SNR fetal brain imaging. In: ISBI 2006, pp. 662-665 (2006)

[6] Jiang, S., et al.: MRI of moving subjects using multi-slice Snapshot images with Volume Reconstruction (SVR): application to fetal, neonatal and adult brain studies. IEEE Tran. Medical Imaging 26(7), 967-980 (2007)

[7] Studholme, C., et al.: An overlap invariant entropy measure of 3D medical image alignment. Pattern Recognition 32(1), 71-86 (1999)

[8] Jenkinson, M., et al.: Improved optimization for the robust and accurate linear registration and motion correction of brain images. NeuroImage 17, 825-841 (2002)

[9] Lee, S., et al.: Scattered data interpolation with multilevel B-splines. IEEE Trans. Visualization Comput. Graph. 3, 228-244 (1997)

[10] Paige, C.C., et al.: LSQR: An Algorithm for Sparse Linear Equations and Sparse Least Squares. ACM Transactions on Mathematical Software (TOMS) 8(1), 43-71 (1982)

[11] Partridge, S.C., et al.: Diffusion tensor imaging: serial quantitation of white matter tract maturity in premature newborns. NeuroImage 22, 1302-1314 (2004) 